\title{
Analytical Treatment and Convergence of the Adomian Decomposition Method for Instability Phenomena Arising during Oil Recovery Process
}

\author{
Ramakanta Meher ${ }^{1,2}$ and Srikanta K. Meher ${ }^{1,2}$ \\ ${ }^{1}$ Department of Mathematics, Sardar Vallabhbhai National Institute of Technology, Surat 395007, India \\ ${ }^{2}$ Department of Mathematics, Anand Agriculture University, Dahod, Gujarat, India
}

Correspondence should be addressed to Ramakanta Meher; meher_ramakanta@yahoo.com

Received 24 February 2013; Revised 7 May 2013; Accepted 13 June 2013

Academic Editor: George S. Dulikravich

Copyright (C) 2013 R. Meher and S. K. Meher. This is an open access article distributed under the Creative Commons Attribution License, which permits unrestricted use, distribution, and reproduction in any medium, provided the original work is properly cited.

An abstract result is proved for the convergence of Adomian decomposition method for partial differential equations that model porous medium equation. Moreover, we prove that this decomposition scheme applied to a porous medium equation arising in instability phenomena in double phase flow through porous media is convergent in a suitable Hilbert space. Furthermore, this technique is utilized to find closed-form solutions for the problem under consideration.

\section{Introduction}

The oil-water movement in a porous medium is an important problem of petroleum technology and water hydrology (Scheidegger [1]). The motion of two immiscible fluids in a homogenous porous medium was obtained by Buckley and Leveret without considering capillary pressure. The basic assumption underlying in the present investigation is that the oil and water form two immiscible liquid phases and water represents preferentially wetting phase.

During secondary oil recovery when a fluid (oil) contained in a porous matrix of an oil formatted region in an oil reservoir is displaced by another fluid of lesser viscosity, that is, water, instead of regular displacement of the whole front, perturberance (fingers) may occur which shoot thorough the porous medium at relatively great speed. These protuberances are called fingers. This phenomenon is called instability. Scheidegger [1] analyzed the statistical behavior of instability in a displacement process through a homogeneous porous medium with capillary pressure and pressure-dependent phase densities. This problem has great importance for oil production in petroleum technology.

Displacement of oil from a porous matrix by an external force which gives rise to a pressure gradient is known as forced instability. Instability can occur in cocurrent and counter-current flow modes.

The stability of a water flood depends on the mobility ratio between oil, water, heterogeneity of the porous medium, segregation of the fluids in the reservoir, and dissipation of fluid fronts caused by capillary pressure. Instabilities may occur in both miscible and immiscible processes and originate on the interface between oil and water. These frontal instabilities are often characterized by a number of penetrating fingers of displacing fluid.

Many of the oil fields around the world contain high viscous oil which gives a mobility ratio greater than unity $(M>1)$. Such mobility ratios are unfavorable and may cause the occurrence of frontal instabilities. If $M>1$, the injected water may channel through the oil in an unstable manner. This viscous fingering leads to premature breakthrough of water and a poor volumetric sweep. In principle, an increase in water viscosity should give lower residual oil saturation due to an increase in the capillary number. This increase is however normally too small to significantly affect the residual oil saturation.

Many researchers have studied this phenomenon with different points of view. The problem of the flow of two immiscible phases in homogeneous porous media without 
capillary pressure effect has been discussed by Buckely and Leverett [2]. Oroveanu [3] has formally extended this discussion for heterogeneous porous media. Verma [4] has discussed the statistical behavior of fingering in a displacement process in heterogeneous porous medium with capillary pressure. Venkateswar [5] has discussed on the flow of immiscible liquids in a heterogeneous porous medium with capillary pressure and connate water saturation.

In this paper, we investigate the applicability of Adomian decomposition method to the nonlinear partial differential equation arising in instability phenomena in double phase flow through porous media in order to obtain the analytical solution.

The paper is organized as follows: in Section 2, we have written the statement of the problem along with some relation. Fundamental equation of instability phenomena is discussed in Section 3. In Section 4, we introduce the Adomian decomposition method applied to solve nonlinear functional equations. The novelty of this paper is in Section 5, where we develop and prove the convergence of Adomian decomposition scheme, which leads to an abstract result, and analytical solution to the equation. Section 6 is devoted to simulation results for some interesting choices of initial data. We conclude by summarizing the paper in Section 7 .

\section{Statement of Problem}

It is well known that in secondary oil recovery process when water with constant velocity " $v$ " is injected into a seam saturated with oil and consisting of homogenous porous medium, it is assumed that the entire oil on the initial boundary of the seam, $x=0$ ( $x$ is measured in the direction of displacement), is displaced through a small distance due to the impact of injecting water which forms instability at the common interface where water meets the oil zone. To understand this phenomenon, we consider here a horizontal porous matrix of length $L$ with its impermeable surface filled with oil formatted porous media.

For the definiteness of the problem, consider that there is a uniform water injection into oil saturated porous matrix of an oil formatted region having homogenous physical characteristics such that the injecting water outs through the oil formation region of the oil reservoir and gives rise to perturberance (fingers) at the interface where injected water pushes the oil from the oil formatted region. This furnishes well-developed fingers as in Figure 1. The stability of a water flood depends on the mobility ratio between oil and water, heterogeneity of the porous medium, segregation of the fluids in the reservoir, and dissipation of fluid fronts caused by capillary pressure. Instabilities may occur in both miscible and immiscible processes and originate on the interface between oil and water. These frontal instabilities are often characterized by a number of penetrating fingers of displacing fluid. Therefore, the entire oil at the initial boundary $x=0$ ( $x$ being measured in the direction of displacement) is displaced through a distance " $L$ " due to water injection. It is further assumed that complete saturation exists at the initial boundary, and the saturation of displaced water (fingers) in oil zone may happen up to distance $x=L$.

2.1. Relative Permeability and Phase Saturation Relation. Following Scheidegger [1], the relationship between relative permeability and phase saturation may be taken as

$$
k_{w}=S_{w}, \quad k_{0}=1-\alpha S_{w} \quad(\alpha=1.11),
$$

where $k_{w}$ and $k_{o}$ denote the relative permeabilities of water and oil and $S_{w}$ be the saturation of water (regarded as the wetting phase).

\section{Fundamental Equations}

The seepage velocity of water $\left(v_{w}\right)$ and oil $\left(v_{o}\right)$ may be written as (by Darcy's law)

$$
v_{o}=-\left(\frac{k_{o}}{\mu_{o}} K\right) \frac{\partial p_{o}}{\partial x} \quad v_{w}=-\left(\frac{k_{w}}{\mu_{w}} K\right) \frac{\partial p_{w}}{\partial x},
$$

where " $K$ " is the permeability of homogenous medium $k_{o}$ and $k_{w}$ are relative permeability of oil and water which are function of $S_{o}$ and $S_{w} . S_{w}$ and $S_{o}$ are saturation of water and oil, respectively, and $p_{w}$ and $p_{o}$ denote pressure of water and oil, while $\mu_{w}, \mu_{o}$ are constant kinematic viscosities of the phases in homogenous porous media.

The equations of continuity (phase densities are regarded as constant throughout the process of instability) are

$$
\phi \frac{\partial S_{w}}{\partial t}+\frac{\partial v_{w}}{\partial x}=0, \quad \phi \frac{\partial S_{o}}{\partial t}+\frac{\partial v_{o}}{\partial x}=0,
$$

where " $\phi$ " is the porosity of the medium.

From the definition of capillary pressure $\left(p_{c}\right)$ and phase saturation " $S_{w}$ ", it is evident that

$$
p_{c}=p_{o}-p_{w}, \quad S_{o}+S_{w}=1
$$

Equation (2)-(4) gives

$$
\phi \frac{\partial S_{w}}{\partial t}+\frac{1}{2} \frac{\partial}{\partial x}\left[K \frac{k_{w}}{\mu_{w}} \frac{d p_{c}}{d S_{w}} \frac{\partial S_{w}}{\partial x}\right]=0 .
$$

For definiteness of the mathematical analysis, we assume standard forms of Verma [4] or the analytical relationship between the relative permeability phase saturation and a linear relationship between capillary pressure phase saturation as

$$
k_{w}=S_{w}, \quad p_{c}=\beta\left(C_{0}+S_{w}^{-2}\right) \quad\left(\beta, C_{0} \text { is constant }\right),
$$

where $k_{w}$ is function of $S_{w}$ only (Scheidegger [1]) and the negative power of the second term is due to the fact that $p_{c}$ is a decreasing function of $S_{w}$.

Substituting this value in (5), we get

$$
\frac{\partial S_{w}}{\partial t}=\frac{K \beta}{\mu_{w} \phi} \frac{\partial}{\partial x}\left(\frac{1}{S_{w}^{2}} \frac{\partial S_{w}}{\partial x}\right) .
$$



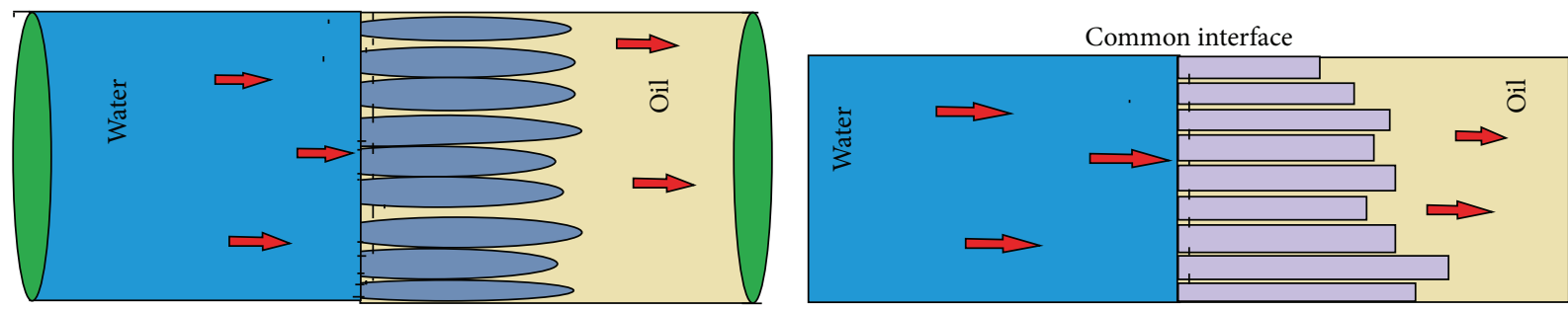

FIGURE 1: Diagram showing the formation of instability of oil zone due to the external force of injected water during secondary recovery in an oil reservoir.

Equation (7) represents a nonlinear partial differential equation describing instability phenomenon of two immiscible fluids (i.e., water and oil) flow through homogeneous porous cylindrical medium with impervious bounding surfaces on three sides of an oil reservoir.

Choosing dimensionless variables $X=x / L, T=$ $\left(K \beta / \phi \mu_{w} L^{2}\right) t$.

It implies if $0<x<L$, then $0<X<1$, and here we will study the behavior of injected water up to $X=1$.

The dimensionless form of (7) is as

$$
\frac{\partial S_{w}}{\partial T}=\frac{\partial}{\partial X}\left(\frac{1}{S_{w}^{2}} \frac{\partial S_{w}}{\partial X}\right)
$$

We choose appropriate initial and Dirichlet's boundary condition due to the behavior of saturation of displaced water at the interface in instability phenomena; that is, instability of oil and water zone at the interface is high, and it becomes stable as it becomes away from the interface $X=0$ and by Verma [4] as

$$
\begin{gathered}
S_{w}(X, 0)=S_{w, 0}(X)=\left(X^{2}+1\right)^{-1 / 2}, \\
S_{w}(0, T)=f_{1}(T), \quad S_{w}(1, T)=f_{2}(T),
\end{gathered}
$$

where $f_{1}$ and $f_{2}$ are the saturation of water at common interface $X=0$ and saturation of water at end of matrix of length $X=1$ (i.e., $x=L$ ). Here, during instability phenomena saturation fingers may take place up to the end of matrix, that is, up to $x=L$. To stabilize or to find the behavior of the saturation fingers, it is necessary to discuss the behavior of saturation of displace water by solving (8) together with (9).

Equation (8) is the desired nonlinear partial differential equation with suitable initial and boundary conditions which describes the saturation of displaced water in instability phenomena arising during oil recovery process.

\section{Analysis of the Adomian Method}

In the early 1980s, a new numerical method was developed by Adomian [6] in order to solve nonlinear functional equations of the form

$$
L S_{w}+R S_{w}+N S_{w}=g
$$

using an iterative decomposition scheme that led to elegant computation of closed-form analytical solutions or analytical approximations to solutions. In (10), $L$ represents the linear part, $N$ represents the nonlinear part, $R$ represents the remainder or lower order terms, and $g$ is the nonhomogeneous right-hand side. The solution $S_{w}$ and the nonlinearity $N$ are assumed to have the following analytic expansions, respectively:

$$
S_{w}=\sum_{n=0}^{\infty} S_{w n}, \quad N S_{w}=\sum_{n=0}^{\infty} A_{n}
$$

where the $A_{n}$ 's are the Adomian polynomials that depend only on $S_{w 0}, S_{w 1}, S_{w 2}, \ldots, S_{w n}$ and are given by the following formula:

$$
A_{n}=\frac{1}{n !} \frac{d^{n}}{d \lambda^{n}}\left[N\left(\sum_{k=0}^{\infty} \lambda^{k} S_{w k}\right)\right]_{\lambda=0}, \quad n \geq 0 .
$$

In order to better explain the method, we will first assume the convergence of the series in (11) and deal with the rigorous convergence issues later. The parameter $k$ is a dummy variable introduced for ease of computation. There are several different versions of (12) that can be found in the literature that leads to easier computation of the $A_{n}$ 's. It should be noted that the $A_{n}$ 's are the terms of analytic expansion of $N S_{w}$, where $S_{w}=\sum_{n=0}^{\infty} S_{w n} \lambda^{n}$.

In [7], Adomian has shown that the expansion for $N S_{w}$ in (11) is a rearrangement of the Taylor series expansion of $N S_{w}$ about the initial function $S_{w 0}$ in a suitable Hilbert or Banach space. Substitution of (11) in (10) results in the following:

$$
L\left(\sum_{n=0}^{\infty} S_{w n}\right)=-R\left(\sum_{n=0}^{\infty} S_{w n}\right)-\sum_{n=0}^{\infty} A_{n}+g .
$$

The above equation can be rewritten in a recursive fashion, yielding iterates $S_{w n}$, the sum of which converges to the solution $S_{w}$ satisfying (13) if it exists

$$
\begin{gathered}
\sum_{n=0}^{\infty} S_{w n}=-L^{-1} R\left(\sum_{n=0}^{\infty} S_{w n}\right)-L^{-1} \sum_{n=0}^{\infty} A_{n}+L^{-1}(g), \\
S_{w 0}=L^{-1}(g) ; \quad S_{w, n+1}=-L^{-1} R\left(S_{w n}\right)-L^{-1}\left(A_{n}\right) .
\end{gathered}
$$

Typically, the symbol $L^{-1}$ represents a formal inverse of the linear operator $L$. In the case of partial differential equations, $L$ is the highest order partial derivative operator for which the formal inverse can be computed using integrations. 
A general theory of decomposition schemes for nonlinear functional equations was developed by Gabet [8]. Convergence results as applied to ordinary differential equations and nonlinear functional equations can be found in $[7,9,10]$. Mavoungou [11] has proved a convergence result for the Adomian scheme as applied to partial differential equations. A compendium of interesting examples of partial differential equations for which the Adomian method was utilized can be found in [12]. In general, the iterates in the Adomian decomposition scheme (14) converge very rapidly to the unique solution of the functional equation (10) provided that the scheme satisfies the property of strong convergence as discussed in [8].

\section{Convergence Analysis of the Adomian Decomposition Method}

We recall the following theorem from [11] which guarantees the convergence of Adomian's method for the general operator equation given by $L S_{w}+R S_{w}+N S_{w}=g$.

Consider the Hilbert space $H=L^{2}((\alpha, \beta) \times[0, T])$ defined by the set of applications:

$$
\begin{aligned}
& S_{w}:(\alpha, \beta) \times[0, T] \longrightarrow R \\
& \text { with } \int_{(\alpha, \beta) \times[0, T]} S_{W}^{2}(\eta, \xi) d \eta d \xi<+\infty .
\end{aligned}
$$

Let us denote

$$
\begin{gathered}
L S_{w}=\frac{\partial S_{w}}{\partial T}, \quad N S_{w}=S_{w}^{-2} \frac{\partial S_{w}}{\partial X}, \\
T S_{w}=R S_{w}+N S_{w}=\frac{\partial}{\partial X}\left(S_{w}^{-2} \frac{\partial S_{w}}{\partial X}\right) .
\end{gathered}
$$

Theorem 1. Let $T S_{w}=-R S_{w}-N S_{w}$ be a hemicontinuous operator in a Hilbert space $H$ and satisfy the following hypothesis:

$\left(H_{1}\right)$

$$
\begin{array}{r}
\left(T S_{w}^{\prime}-T S_{w}^{* \prime}, S_{w}^{\prime}-S_{w}^{* \prime}\right) \geq k\left\|S_{w}^{\prime}-S_{w}^{* \prime}\right\|^{2}, \\
k>0, \forall S_{w}^{\prime}, S_{w}^{*^{\prime}} \in H,
\end{array}
$$

$\left(H_{2}\right)$ whatever may be $M>0$, there exist constant $C(M)>0$ such that for $S_{w}^{\prime}, S_{w}^{*} \in H$ with $\left\|S_{w}^{\prime}\right\| \leq M,\left\|S_{w}^{* \prime}\right\| \leq M$, we have

$$
\begin{array}{r}
\left(T S_{w}^{\prime}-T S_{w}^{* \prime}, w\right) \leq C(M)\left\|S_{w}^{\prime}-S_{w}^{* \prime}\right\|\|w\| \\
\text { for every } w \in H .
\end{array}
$$

Then, for every $g \in H^{\prime}$, the nonlinear functional equation $L S_{w}+R S_{w}+N S_{w}=g$ admits a unique solution $S_{w} \in H$. Furthermore, if the solution $S_{w}$ can be represented in a series form given by $S_{w}=\sum_{n=0}^{\infty} S_{w n} \lambda^{n}$, then the Adomian decomposition scheme corresponding to the functional equation under consideration converges strongly to a $S_{w} \in H$, which is the unique solution to the functional equation.
Proof. Verification of hypothesis $\left(H_{1}\right)$

$$
\begin{gathered}
T S_{w}^{\prime}-T S_{w}^{*^{\prime}}=-\frac{\partial^{2}}{\partial X^{2}}\left(S_{w}^{-1^{\prime}}-S_{w}^{-1^{\prime}}\right) \\
\left(T S_{w}^{\prime}-T S_{w}^{* \prime}, S_{w}^{\prime}-S_{w}^{* \prime}\right) \\
\quad=\left(-\frac{\partial^{2}}{\partial X^{2}}\left(S_{w}^{-1^{\prime}}-S_{w}^{-1 *^{\prime}}\right), S_{w}^{\prime}-S_{w}^{*^{\prime}}\right) .
\end{gathered}
$$

Since $\partial^{2} / \partial X^{2}$ is a differential operator in $H$, then there exist constant " $\delta$ " such that,

according to Schwartz inequality, we get

$$
\begin{gathered}
\left(\frac{\partial^{2}}{\partial X^{2}}\left(S_{w}^{-1 \prime}-S_{w}^{-1 * \prime}\right), S_{w}^{\prime}-S_{w}^{* \prime}\right) \\
\quad \leq \delta\left\|S_{w}^{-1^{\prime}}-S_{w}^{-1 *^{\prime}}\right\|\left\|S_{w}^{\prime}-S_{w}^{*^{\prime}}\right\| .
\end{gathered}
$$

Now, we use mean value theorem, then we have

$$
\begin{aligned}
& \left(\frac{\partial^{2}}{\partial X^{2}}\left(S_{w}^{-1 \prime}-S_{w}^{-1 * \prime}\right), S_{w}^{\prime}-S_{w}^{* \prime}\right) \\
& \quad \leq \delta\left\|S_{w}^{-1^{\prime}}-S_{w}^{-1 *^{\prime}}\right\|\left\|S_{w}^{\prime}-S_{w}^{*^{\prime}}\right\| \leq \frac{\delta}{M^{2}}\left\|S_{w}^{\prime}-S_{w}^{*^{\prime}}\right\|^{2},
\end{aligned}
$$

for $\left\|S_{w}^{\prime}\right\| \leq M$ and $\left\|S_{w}^{* \prime}\right\| \leq M$.

Therefore,

$$
\left(-\frac{\partial^{2}}{\partial X^{2}}\left(S_{w}^{-1 \prime}-S_{w}^{-1 * \prime}\right), S_{w}^{\prime}-S_{w}^{* \prime}\right) \geq \frac{\delta}{M^{2}}\left\|S_{w}^{\prime}-S_{w}^{*^{\prime}}\right\|^{2} .
$$

Substituting (22) in (19),

$$
\left(T S_{w}^{\prime}-T S_{w}^{* \prime}, S_{w}^{\prime}-S_{w}^{* \prime}\right) \geq k\left\|S_{w}^{\prime}-S_{w}^{* \prime}\right\|^{2},
$$

where $k=\delta / M^{2}$. Hence we find the hypothesis $\left(H_{1}\right)$.

For hypothesis $\left(\mathrm{H}_{2}\right)$,

$$
\begin{aligned}
\left(T S_{w}^{\prime}-T S_{w}^{* \prime}, V\right) & =\left(-\frac{\partial^{2}}{\partial X^{2}}\left(S_{w}^{-1 \prime}-S_{w}^{-1 * \prime}\right), V\right) \\
& \leq \frac{1}{M^{2}}\left\|S_{w}-S_{w}^{* \prime}\right\|\|V\| \\
& =C(M)\left\|S_{w}-S_{w}^{*^{\prime}}\right\|\|V\|,
\end{aligned}
$$

where $C(M)=1 / M^{2}$, and, therefore, $\left(H_{2}\right)$ holds. The proof is complete.

Remark 2. We note that the constant $C(M)$ is function of $M$, and the linearity of $T$ allows us to prove $\left(\mathrm{H}_{2}\right)$. Furthermore, since every linear continuous operator is hemicontinuous, the operator $T$ is hemicontinuous.

\section{Simulations Results}

Using the analysis of Adomian Decomposition Method, (8) can be written in operator form $L_{T} S_{w}$ as

$$
L_{T} S_{w}(X, T)=L_{X}\left(N S_{w}(X, T)\right) .
$$


Operating the inverse operators on both sides of (25), it gives

$$
S_{w}(X, T)=S_{w 0}(X)+L_{T}^{-1}\left[L_{X}\left(N S_{w}(X, T)\right)\right]
$$

where $N S_{w}(X, T)=S_{w}\left(\partial S_{w} / \partial X\right)$ and $S_{w 0}(X)$ can be solved subject to the corresponding initial condition (9).

It is well known from (11) that the solution of (8) can be written in series form as follows:

$$
S_{w}(X, T)=\sum_{n=0}^{\infty} S_{w n}(X, T)
$$

where $S_{w 0}, S_{w 1}, S_{w 2}, \ldots$ are the saturations of different fingers at any distance $X$ and an time $T>0$ and the nonlinear term can be represented as $N S_{w}(X, T)=\sum_{n=0}^{\infty} A_{n}$, where $A_{n}$ 's are the Adomian's special polynomials to be determined and defined by (12).

Following the analysis of Adomian decomposition method as discussed in $[7,8]$, for the determination of the components $S_{w, n}(X, T)$ of $S_{w}(X, T)$, we set the recursive relation as

$$
\sum_{n=0}^{\infty} S_{w, n}(X, T)=\left(X^{2}+1\right)^{-1 / 2}+L_{T}^{-1}\left[L_{X}\left(\sum_{n=0}^{\infty} A_{n}\right)\right],
$$

where $S_{w 0}=\left(X^{2}+1\right)^{-1 / 2}=S_{w}(X, 0)$ from $(9)$ and $S_{w, k+1}=$ $L_{T}^{-1}\left(\left(A_{k}\right)_{x}\right)$.

\subsection{Adomian Polynomials Are as Follows}

$$
\begin{gathered}
A_{0}=S_{w, 0}^{-2}\left(S_{w, 0}\right)_{X} \\
A_{1}=S_{w, 0}^{-2}\left(S_{w, 1}\right)_{X}-2 S_{w, 0}^{-3} S_{w, 1}\left(S_{w, 0}\right)_{X} \\
A_{2}=S_{w, 0}^{-2}\left(S_{w, 2}\right)_{X}-2 S_{w, 0}^{-3} S_{w, 1}\left(S_{w, 1}\right)_{X} \\
-2 S_{w, 0}^{-3} S_{w, 2}\left(S_{w, 0}\right)_{X}+3 S_{w, 0}^{-4} S_{w, 1}^{2}\left(S_{w, 0}\right)_{X}
\end{gathered}
$$

On Substituting (29) into (28), it gives the components of saturation of water as

$$
\begin{gathered}
S_{w, 0}(X, T)=\left(X^{2}+1\right)^{-1 / 2} \\
S_{w, 1}(X, T)=L_{T}^{-1}\left(\left(A_{0}\right)_{X}\right)=-\left(X^{2}+1\right)^{-3 / 2} T
\end{gathered}
$$

$$
\begin{aligned}
S_{w, 2}(X, T) & =L_{T}^{-1}\left(\left(A_{1}\right)_{X}\right)=\left(X^{2}+1\right)^{-5 / 2}\left(1-2 X^{2}\right) \frac{T^{2}}{2 !} \\
S_{w, 3}(X, T) & =L_{T}^{-1}\left(\left(A_{2}\right)_{X}\right) \\
& =-\left(X^{2}+1\right)^{-7 / 2}\left(1-10 X^{2}+4 X^{4}\right) \frac{T^{3}}{3 !}
\end{aligned}
$$

and so on.

In view of (30), the approximate solution in a series form is given by

$$
\begin{aligned}
\sigma_{n}(X, T) & \\
= & \left(X^{2}+1\right)^{-1 / 2} \\
& -\left(X^{2}+1\right)^{-3 / 2} T+\left(X^{2}+1\right)^{-5 / 2}\left(1-2 X^{2}\right) \frac{T^{2}}{2 !} \\
& -\left(X^{2}+1\right)^{-7 / 2}\left(1-10 X^{2}+4 X^{4}\right) \frac{T^{3}}{3 !}+\cdots,
\end{aligned}
$$

which converges to $S_{w}(X, T)$, and the closed-form solution of (8) as

$$
S_{w}(X, T)=\left(X^{2}+e^{2 T}\right)^{-1 / 2} .
$$

It is interesting to note that $S_{w}(X, T)$ in (32) has the following asymptotic behavior:

$$
\begin{aligned}
& \lim _{X \rightarrow \infty} S_{w}(X, T)=0, \\
& \lim _{T \rightarrow \infty} S_{w}(X, T)=0,
\end{aligned}
$$

and the flux satisfying

$$
\lim _{X \rightarrow \infty} S_{w}^{-2}\left(S_{w}\right)_{X}=-1
$$

implies that the saturation of water decreases with distance and time increases. Moreover, this solution vanishes as $X$ approaches infinity.

An explicit general exact solution of the form

$$
S_{w}(X, T)=C^{1 / 2}\left(C^{2}(X+B)^{2}+A e^{2 C T}\right)^{-1 / 2}
$$

by using a generalized conditional symmetry method, where $A, B$, and $C>0$ are arbitrary constants. Moreover, an implicit solution was obtained for $C<0$.

\section{Conclusion}

In this paper, we have proved the convergence of the Adomian decomposition scheme for the case of instability phenomena. The solution (31) of (8) gives the saturation of water $S_{w}(X, T)$ as a function of distance and time in displaced oil zone 
TABLE 1: Saturation versus time keeping distance fixed: Adomian decomposition method.

\begin{tabular}{lcccccccccc}
\hline$X / T$ & 0.1 & 0.2 & 0.3 & 0.4 & 0.5 & 0.6 & 0.7 & 0.8 & 0.9 & 1 \\
\hline 0.1 & 0.901153 & 0.815959 & 0.738585 & 0.668161 & 0.603819 & 0.544687 & 0.489897 & 0.43858 & 0.389865 & 0.342883 \\
0.2 & 0.890376 & 0.807984 & 0.732875 & 0.664522 & 0.602394 & 0.545964 & 0.494703 & 0.448083 & 0.405574 & 0.366648 \\
0.3 & 0.873241 & 0.795169 & 0.723512 & 0.658171 & 0.59905 & 0.54605 & 0.499073 & 0.458021 & 0.422796 & 0.393301 \\
0.4 & 0.850831 & 0.778173 & 0.7108 & 0.649006 & 0.593088 & 0.543343 & 0.500065 & 0.463552 & 0.434098 & 0.412001 \\
0.5 & 0.8244 & 0.757806 & 0.69522 & 0.637214 & 0.584359 & 0.537229 & 0.496395 & 0.462431 & 0.435908 & 0.417399 \\
0.6 & 0.795209 & 0.734933 & 0.677375 & 0.623243 & 0.573248 & 0.528099 & 0.488505 & 0.455177 & 0.428824 & 0.410155 \\
0.7 & 0.764408 & 0.710386 & 0.657894 & 0.60766 & 0.560411 & 0.516877 & 0.477784 & 0.443861 & 0.415836 & 0.394437 \\
0.8 & 0.732959 & 0.684903 & 0.637365 & 0.591013 & 0.546511 & 0.504525 & 0.465723 & 0.430769 & 0.400329 & 0.375069 \\
0.9 & 0.701618 & 0.659097 & 0.616291 & 0.573761 & 0.532069 & 0.491775 & 0.45344 & 0.417626 & 0.384893 & 0.355803 \\
1 & 0.670941 & 0.63345 & 0.595075 & 0.556257 & 0.51744 & 0.479065 & 0.441573 & 0.405408 & 0.37101 & 0.338822 \\
\hline
\end{tabular}

TABLE 2: Generalized conditional symmetry method.

\begin{tabular}{lcccccccccc}
\hline$X / T$ & 0.1 & 0.2 & 0.3 & 0.4 & 0.5 & 0.6 & 0.7 & 0.8 & 0.9 & 1 \\
\hline 0.1 & 0.901156 & 0.816 & 0.738794 & 0.668819 & 0.605418 & 0.547987 & 0.495974 & 0.448876 & 0.406234 & 0.367631 \\
0.2 & 0.890375 & 0.80797 & 0.732818 & 0.664376 & 0.602117 & 0.545535 & 0.494154 & 0.447526 & 0.405232 & 0.366888 \\
0.3 & 0.873237 & 0.795098 & 0.723174 & 0.657164 & 0.596732 & 0.541521 & 0.491165 & 0.445301 & 0.403579 & 0.365659 \\
0.4 & 0.850824 & 0.778069 & 0.710289 & 0.64745 & 0.589432 & 0.536047 & 0.487069 & 0.442243 & 0.401298 & 0.36396 \\
0.5 & 0.824393 & 0.757701 & 0.694693 & 0.635572 & 0.580427 & 0.529247 & 0.481951 & 0.438401 & 0.398421 & 0.36181 \\
0.6 & 0.795204 & 0.734852 & 0.676957 & 0.621905 & 0.569962 & 0.521278 & 0.47591 & 0.433839 & 0.394987 & 0.359232 \\
0.7 & 0.764406 & 0.710342 & 0.65765 & 0.606837 & 0.558295 & 0.512308 & 0.469055 & 0.428627 & 0.391041 & 0.356257 \\
0.8 & 0.732959 & 0.684895 & 0.637302 & 0.59074 & 0.545684 & 0.502511 & 0.461501 & 0.42284 & 0.386633 & 0.352913 \\
0.9 & 0.70162 & 0.659119 & 0.616378 & 0.57396 & 0.532376 & 0.492061 & 0.453367 & 0.416557 & 0.381812 & 0.349235 \\
1 & 0.670944 & 0.633492 & 0.595268 & 0.556799 & 0.518596 & 0.481119 & 0.444765 & 0.409856 & 0.376631 & 0.345258 \\
\hline
\end{tabular}

causing instability at the interface up to distance $X=1$ due to an external force of injected water through injecting well during oil recovery process. Equation (31) is an approximate solution containing four terms of an infinite series. The solution contains negative power terms which shows that the saturation of displaced water, that is instability of fingers decreases as the distance $X$ from the interface increases, and it is obvious for the instability phenomena. The instability of oil and water zone is the maximum at the interface $X=0$ and becomes stable as it away from the interface $X=0$, that is, likely to be stable at the boundary point $X=1$. The absolute errors for saturation of water $S_{w}$ have been calculated. As seen from Tables 1 and 2, Adomian decomposition method gives a very good approximation to the partial exact solution by using only three terms of the decomposition series.

\section{References}

[1] A. E. Scheidegger, The Physics of Flow Through Porous Media, University of Toronto Press, 1960.

[2] S. E. Buckley and M. C. Leverett, "Mechanism of fluid displacement in sands," Transactions of the American Institute of Mining, vol. 146, pp. 107-110, 1942.

[3] T. Oroveanu, "Scurgerea fluiidelor prin medii poroase neomogene," Editura Academiei Republicii Populare Romine, vol. 92, no. $328,1963$.

[4] A. P. Verma, "Statistical behavior of fingering in a displacement process in heterogeneous porous medium with capillary pressure," Canadian Journal of Physics, vol. 47, no. 319, 1969.
[5] G. Venkateswar, "On the flow of immiscible liquids in a heterogeneous porous medium with capillary pressure and connate water saturation," International Journal of Engineering Science, vol. 13, pp. 161-171, 1975.

[6] G. Adomian, Solving Frontier Problems in Physics: the Decomposition Method, Kluwer Academic, Dordrecht, The Netherlands, 1994.

[7] K. Abbaoui and Y. Cherruault, "Convergence of Adomian's method applied to differential equations," Computers and Mathematics with Applications, vol. 28, no. 5, pp. 103-109, 1994.

[8] L. Gabet, "The theoretical foundation of the Adomian method," Computers and Mathematics with Applications, vol. 27, no. 12, pp. 41-52, 1994.

[9] Y. Cherruault, "Convergence of Adomian's method," Kybemetes, vol. 18, no. 2, pp. 31-38, 1989.

[10] Y. Cherruault and G. Adomian, "Decomposition methods: a new proof of convergence," Mathematical and Computer Modelling, vol. 18, no. 12, pp. 103-106, 1993.

[11] T. Mavoungou, "Convergence of Adomian's method and applications to non-linear partial differential equations," Kybernetes, vol. 21, no. 6, pp. 13-25, 1994.

[12] A. M. Wazwaz, Partial Differential Equations: Methods and Applications, Balkema, Tokyo, Japan, 2002. 


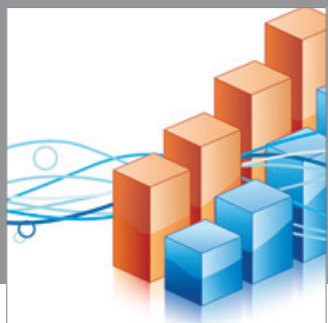

Advances in

Operations Research

mansans

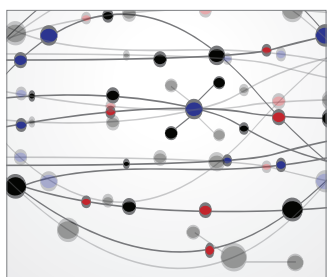

The Scientific World Journal
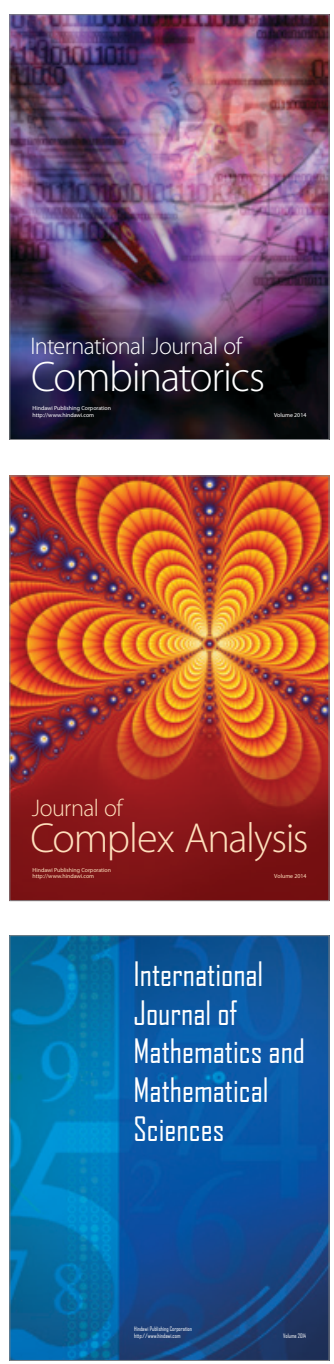
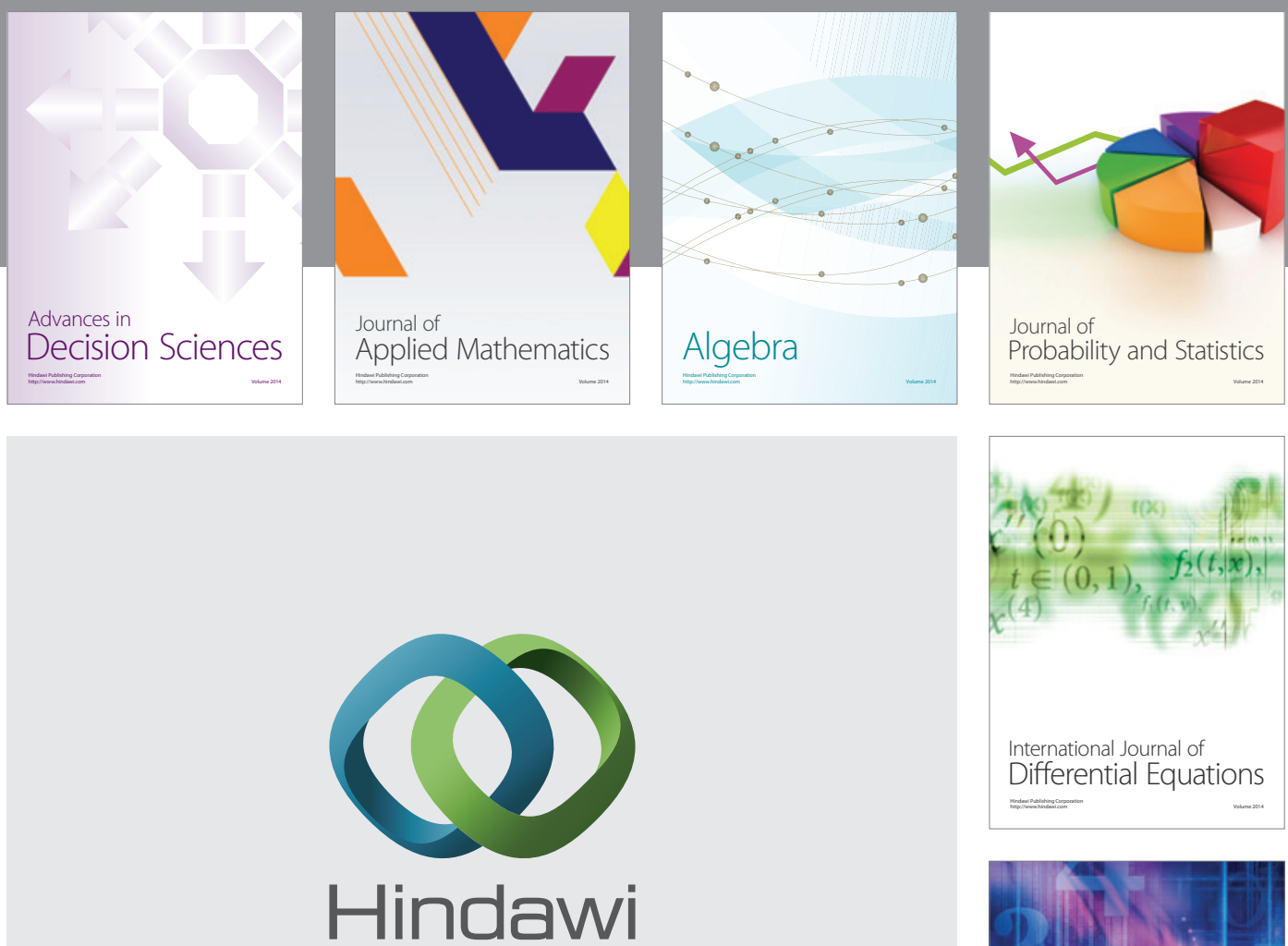

Submit your manuscripts at http://www.hindawi.com
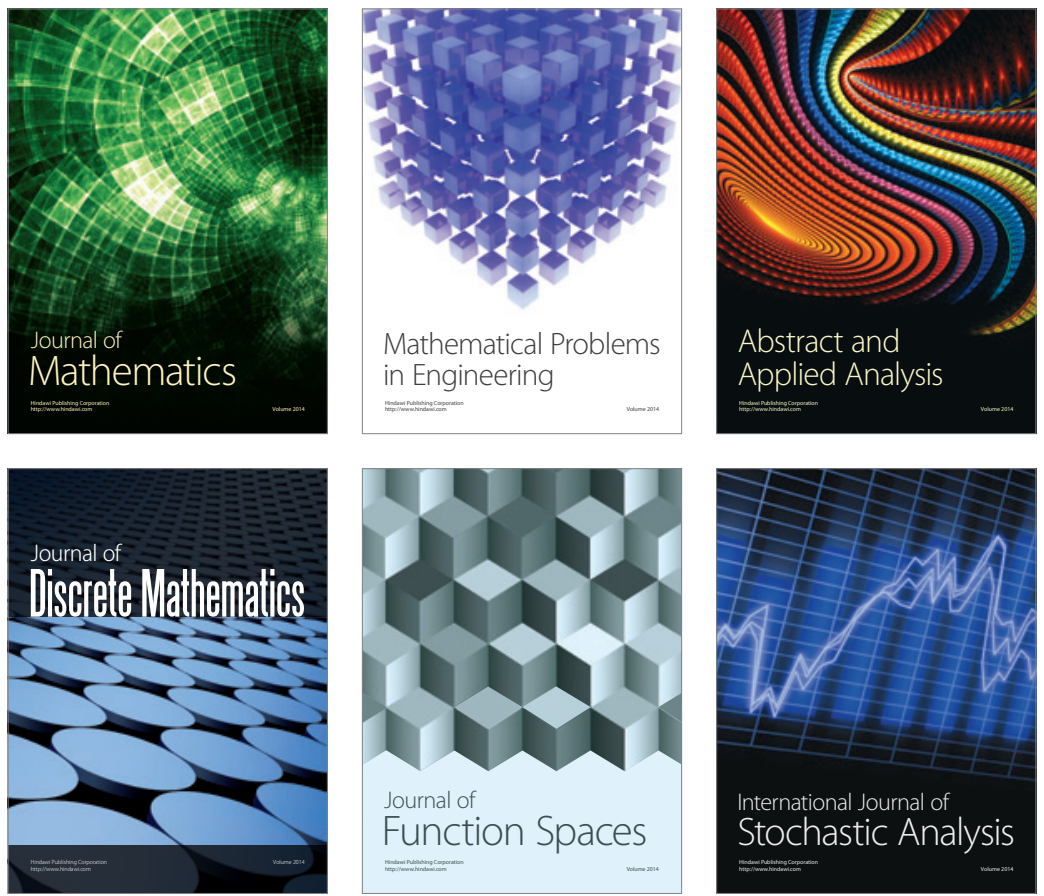

Journal of

Function Spaces

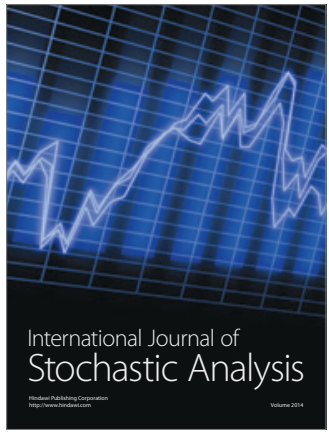

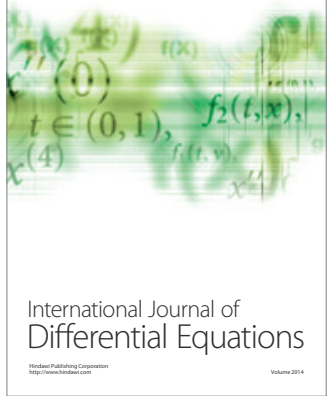
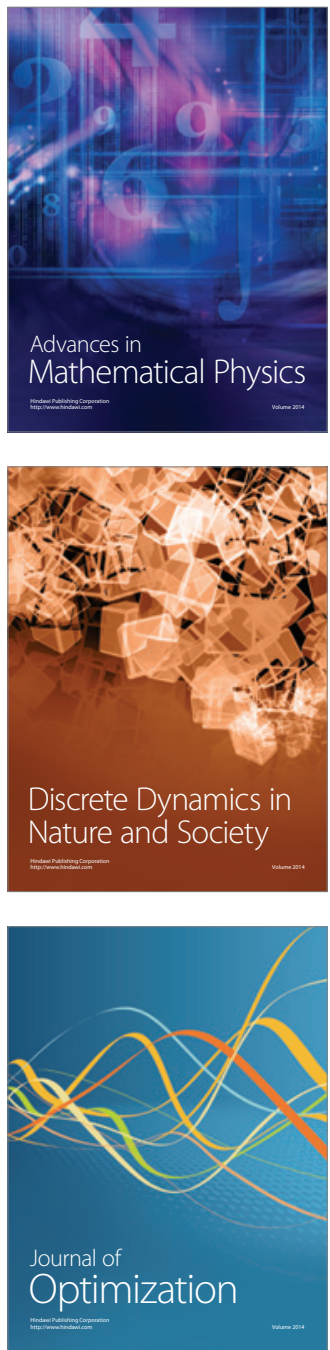